\title{
The European Health Data Space: a step towards digital and integrated care systems
}

\author{
Stefano Genovese \\ Milan Cardiological Centre, Milan, UK \\ Rafael Bengoa \\ Bilbao Institute for Health and Strategy, Bilbao, Spain \\ John Bowis and Mary Harney \\ EGIDE, London, UK \\ Bastian Hauck \\ International Diabetes Federation Europe, Berlin, Germany \\ Michel Pinget \\ CEED Diabetes, Paris, France \\ Mike Leers \\ EGIDE, London, UK \\ Tarja Stenvall \\ Sanofi SA, Paris, France, and \\ Nick Guldemond \\ Department of Public Health and Primary Care, \\ Leiden Universitair Medisch Centrum, Leiden, The Netherlands and \\ Department N.A. Semashko, Institute of Public Health F.F. Erisman, \\ I.M. Sechenov First Moscow State Medical University, Moscow, Russian Federation
}

\begin{abstract}
Purpose - The COVID-19 pandemic has demonstrated the urgency of better chronic disease management and the importance of making it an integral part of the recovery agenda in Europe. This paper aims to explore the shift towards digital and integrated care systems in Europe.

Design/methodology/approach - In this viewpoint paper the Expert Group for Integrated Care and Digital Health Europe (EGIDE) group argues that an orchestrated shift towards integrated care holds the solution to the chronic disease pandemic.

Findings - The development of integrated care cannot happen without shifting towards a digitalised healthcare system via large-scale initiatives like the European Health Data Space (EHDS) and the involvement of all stakeholders.
\end{abstract}

(C) Stefano Genovese, Rafael Bengoa, John Bowis, Mary Harney, Bastian Hauck, Michel Pinget, Mike Leers, Tarja Stenvall and Nick Guldemond. Published by Emerald Publishing Limited. This article is published under the Creative Commons Attribution (CC BY 4.0) licence. Anyone may reproduce, distribute, translate and create derivative works of this article (for both commercial and non-commercial purposes), subject to full attribution to the original publication and authors. The full terms of this licence may be seen at http://creativecommons.org/licences/by/4.0/legalcode

EGIDE is financially supported by Sanofi, however each group member retains full independence and ownership of the views and recommendations shared in this paper. Group members contributed to this policy paper in their personal capacity.

Digital and integrated care systems 
JICA

30,4

Originality/value - The EGIDE group has identified some foundational principles, which can guide the way to realise the full potential of the EHDS for integrated care and can support the involved stakeholders' thinking.

Keywords Integration, Chronic care, Health systems, COVID 19, Disease management, EGIDE group Paper type Viewpoint

\section{Introduction}

This reflection paper aims to represent the views of the chronic disease community. It provides a unique combination of perspectives from academia, healthcare professionals (HCPs), patients and industry. It comes after the first paper, presented by the Expert Group for Integrated Care and Digital Health Europe (EGIDE) in 2020, "Putting people at the centre: Integrated care for chronic diseases in Europe" which reflected on the importance of prioritising the shift towards integrated care models in Europe and the role, which policy-makers have to play in this.

In this paper, the authors acknowledge that too many patients across Europe are experiencing fragmented and delayed care. Covid-19 has highlighted the weaknesses of our healthcare systems and the need for rapid transformation. The European Health Data Space (EHDS) can act as a catalyst for change in several important areas:

(1) Approach to health data from an integrated care perspective:

- How to accelerate data integration?

- What data governance framework is needed to ensure interoperability both within and between health systems?

- How to recognise the value of health data?

- What does it take to use health data to improve patient care and enable self-care?

- How should we reconcile individual patient data privacy with collective data research?

(2) Digital health financing:

- How to guide new investment in data infrastructure?

- What are the new reimbursement and financing models needed to facilitate integrated digital healthcare?

- What are the appropriate financial incentives for integrated digital care? And how to target funds to strengthen healthcare digital infrastructure, given the varying organisation of healthcare systems?

(3) Putting patients in the centre of the new organisation of healthcare systems:

- How to enhance patients' trust in health data?

- How to ensure patients' access to digital health innovation?

(4) Digital health education as an enabler of integrated care:

- How to improve digital health education and training for HCPs?

- How to improve patient education and understanding of the purpose and methods of digital health?

While those topics are at the core of the future European Union legislative cycle, this paper will go a step further. The time of accelerated change in healthcare, which we now live in, offers an unprecedented opportunity not only to shift but also to broaden our thinking around the holistic healthcare system organisational model. EU and national policy makers will have an important role to play in embracing and driving an orchestrated change. 
This reflection paper aims to serve as a starter of a constructive dialogue at various levels. It will provide value to the stakeholders who are advancing and benefiting from theEHDS initiative and who are looking at the re-organisation of the healthcare systems in Europe in general patients, HCPs , EU and national policymakers, digital infrastructure players, EU and national implementing bodies, national insurers and the healthcare payer and research community.

\section{Context: why action on integrated care is urgently needed? Chronic diseases snapshot}

According to the World Health Organisation (WHO), chronic diseases kill 41 million people each year, equivalent to $71 \%$ of all deaths globally. Cardiovascular diseases account for most NCD deaths, or 17.9 million people annually, followed by cancers (9.3 million), respiratory diseases (4.1 million) and diabetes (1.5 million) [1]. This represents a significant social and economic burden to the European society, too. In this context, countries are developing new approaches to chronic disease management to address the requirements associated with chronic conditions and to provide appropriate support to people living with them [2].

\section{The COVID-19 pandemic and chronic diseases}

The COVID-19 pandemic has demonstrated the urgency of better chronic disease management and the importance of making it an integral part of the recovery agenda in Europe. Chronic diseases have proven to be major risk factors for COVID-19 and increase patients' vulnerability, increasing mortality rates (in some countries, over $90 \%$ of hospitalised patients who did not survive had accompanying chronic diseases [3]). During the pandemic, it has become ever more evident how crucial it is to have the right healthcare systems set-up to provide digital solutions like telemonitoring (e.g., condition tracking and risk prediction) \& remote management (e.g., remote administration of medications and therapies). Such solutions would not be possible without the seamless generation and access to high quality, interoperable, integrated health data (see Figure 1).

The pandemic has, therefore, highlighted various challenges of the healthcare systems in Europe when it comes to chronic disease care. The WHO emphasises the crucial role of prevention and control of chronic disease, to mitigate future health crises [4] The EU is focussing more and more on ways to address the chronic disease challenge sustainably by concentrating on risk factors rather than consequences. Thus, there are large investments under the EU health programme as well as investments into research channelled through the health cluster of horizon Europe. Further evidence of this is seen both In the extension of the European Centre for Disease Prevention and Control (ECDC) mandate to reinforce chronic disease prevention and management and in the focus on setting up a comprehensive interoperable health data system through the EHDS.

\section{Integrated care as a solution for chronic diseases}

Clearly, for chronic diseases to be controlled, an integrated approach, involving all stakeholders is needed [5]. Integrated care, as defined by the EGIDE in 2020 [6], involves the provision of seamless, effective and efficient care and prevention that responds to all of a person's health, social and personal needs. This spans physical, social and mental health, in partnership with the individuals, their caregivers and family (see Figure 2).

The development of integrated care cannot happen without shifting towards a digitalised healthcare system. Now is the right time to rethink healthcare systems in Europe, using the momentum of the COVID-19 pandemic and the inevitable and accelerating digitalisation in health, precisely through initiatives like the EHDS.

This shift needs to be guided by:

(1) A comprehensive and collaborative perspective to improve healthcare practises;

\section{Digital and} integrated care systems 
JICA

30,4

366

Figure 1.

Pandemic

preparedness: Tackle

root vulnerabilities

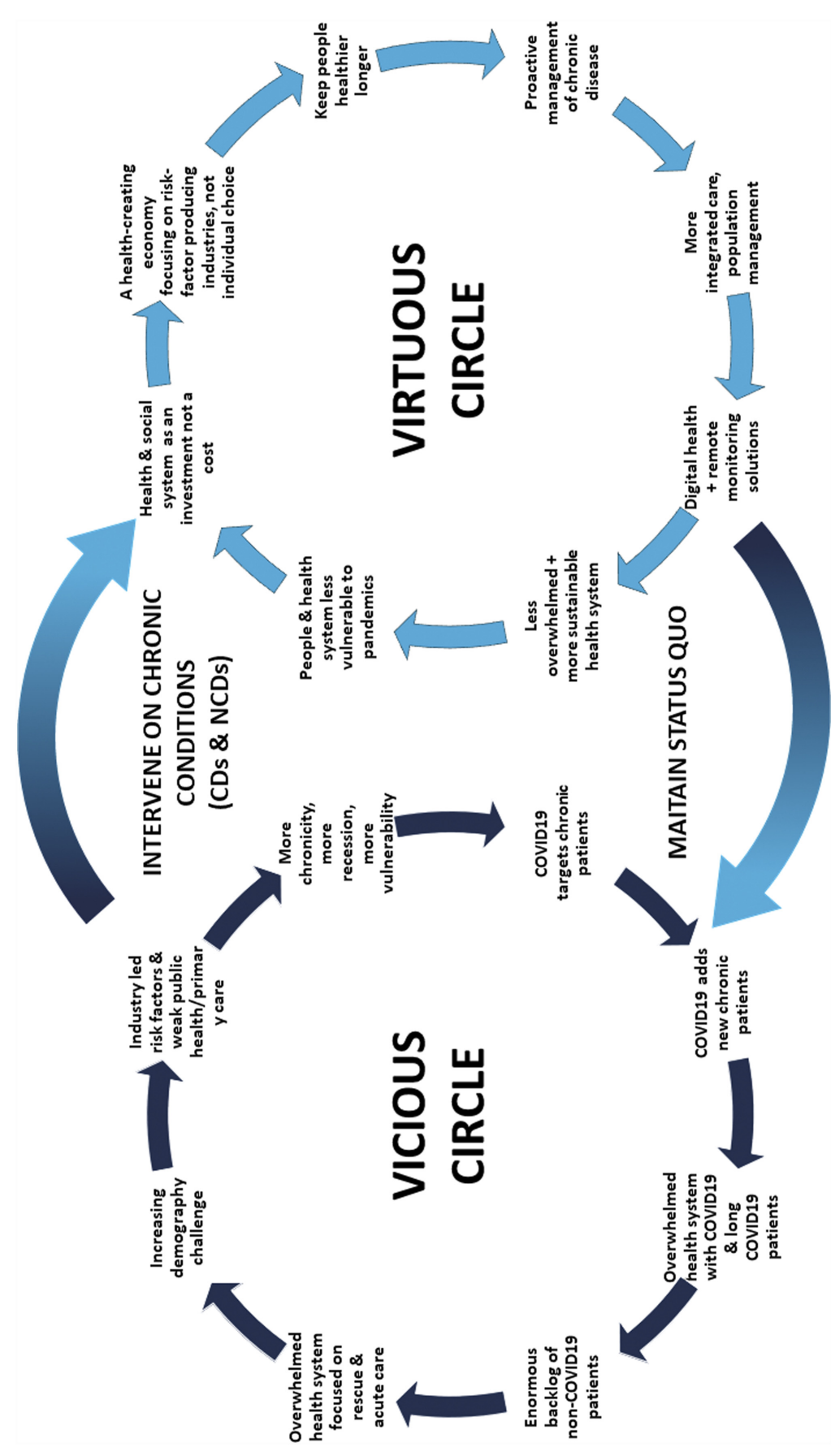




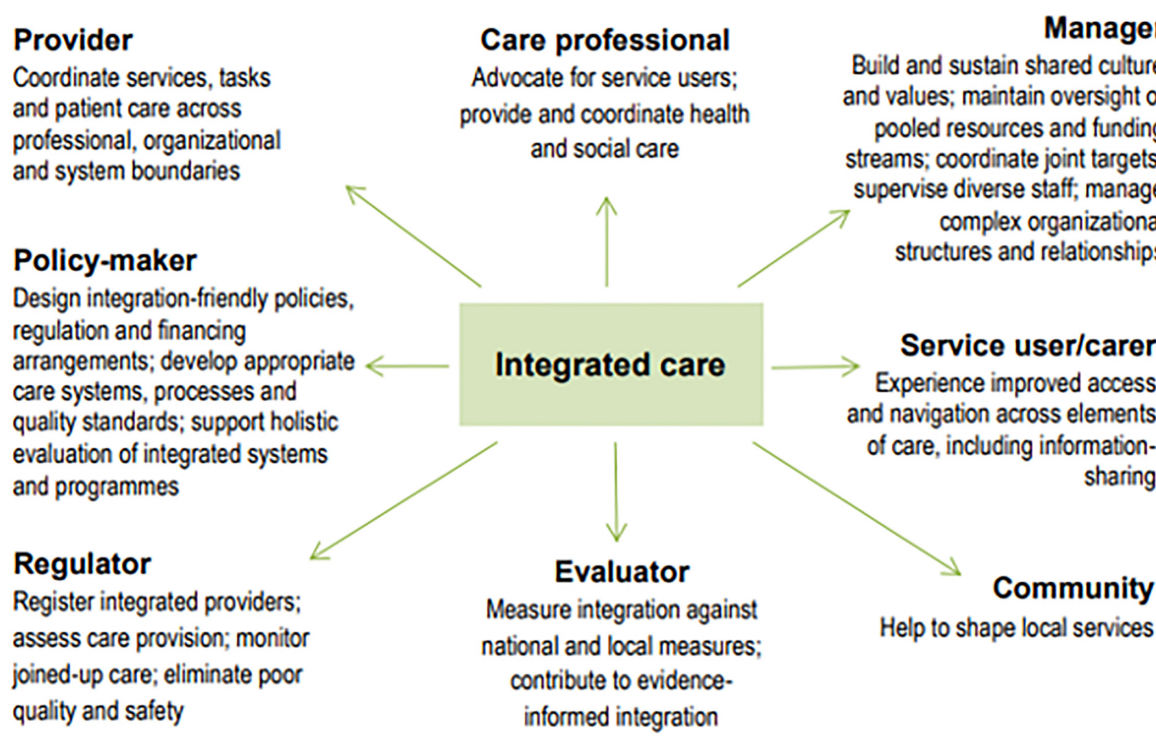

Digital and
integrated care
systems
$\mathbf{3 6 7}$

(2) The needs of patients to manage their condition alongside the healthcare system;

(3) The involvement of stakeholders at all levels and areas of healthcare.

Integrated care and digitalisation then has the potential to:

(1) Dramatically improve the lives of patients living with chronic diseases;

(2) Facilitate the work of HCPs;

(3) Provide optimisation of resources and efficiencies for the healthcare systems in general.

The opportunity ahead of the European Health Data Space for chronic diseases in Europe

A strong EU commitment

The current European Commission 2019-2025, from the start of its mandate, has shown a strong commitment to the establishment of a general European Data Space with more specific focus on several strategic sectors, healthcare being one of the main priorities. A common EHDS will be built on the basis of strong data governance, data quality and interoperability. It aims to promote greater exchange and access to different types of health data (electronic health records, genomics data, data from patient registries etc.). This will not only support healthcare delivery (so-called primary use of data) but also will enhance health research and health policy-making (so-called secondary use of data) [7].

While the European Commission is draughting the final legislation to lay the foundations of the framework, the EU member states will play a key role in the development and implementation of the EHDS, through the "Joint Action for the European Health Data Space". EGIDE firmly believes in the instrumental role of member states and acknowledges that such a large scale and important effort needs not only to be concerted, but also supported and accelerated by each player individually. 
JICA

30,4

368

The potential of the European Health Data Space for integrated care

If developed and implemented with the appropriate principles in its vision, the EHDS has the potential not only to contribute but to be the foundation for a truly integrated healthcare system in Europe by bringing benefits for:

(1) The holistic management of chronic diseases

The availability and access to data can help improve diagnostic timelines and facilitate remote monitoring to ease chronic conditions. Importantly, with the power of data analytics and predictive modelling, the opportunities ahead of prevention and early detection have never been so vast. Chronic diseases are often mismanaged due to the lack of personalised approach. The data available through the EHDS can enable the development of personalised medicine and precision treatments by fostering the possibility to match the patient's genetic characteristics to the most effective treatment solution based on a data analysis.

The holistic management of chronic diseases is crucial not only for those patients themselves, but for the whole healthcare system and society in general.

(2) The optimisation of healthcare systems and the work of HCPs and general improvement of healthcare organisation

Better resource utilisation can be achieved through greater efficiency and optimisation in healthcare delivery. The EHDS can enable predictive techniques, which will improve the efficiency of patient operational flow - helping to prioritise activities within hospitals, improve hospitals' ability to admit patients and accelerating patient discharges, while delivering a more positive patient experience [8].

Problems related to access to health services and shortage of HCPs can be alleviated by the use of health data to feed algorithms (such as artificial intelligence systems) to perform time-consuming tasks faster and with a greater precision, thus supporting HCPs and optimising resource usage (Topol, 2019). Algorithms can reduce the administrative burden currently placed on the work of HCPs by extracting relevant information from patient health records, thereby facilitating staff to increase their face time with patients (Hazarika, 2020). In addition to reducing occupational stress from a high workload, AI tools can also aid HCPs by enhancing accurate diagnoses and reducing medical errors. The EHDS will further foster these positive developments by ensuring that all active stakeholders in the health sector have the capacity to keep an electronic up-to-date health record, ensuring that all important information is easily accessible.

(3) The achievement of a higher quality of life for patients

All this can fit into the achievement of a better life for patients. An improved chronicdisease management will empower patients to self-manage their conditions, and as much as possible to prevent their development, with the help of remote and real-time monitoring via integrated health data systems. Consequently, it will bring a greater quality and equality of their outcomes and lead to an improved result not only for patients, but also for carers, HCPs, healthcare systems and the society and economy in general.

Moreover, the availability of high quality and integrated health data in Europe, if managed correctly, will attribute to the development of new innovative treatments through the secondary use of data, as well as personalised and precision treatments (even across the border) addressing general and rare diseases. 


\section{How to build a European Health Data Space for a truly integrated healthcare system \\ Foundational principles}

The EGIDE group has identified some foundational principles, which can guide the way to realise the full potential of the EHDS for integrated care and can support the stakeholders in their thinking.

EGIDE would be delighted to have a discussion with stakeholders on the following principles, that we consider essential to the reorganisation of the healthcare systems in Europe and, consequently, the development of integrated care:

\section{(1) Policy flexibility}

The EHDS will provide an innovative, transformational framework for the reform of healthcare systems. It will provide the ground for existing and emerging digital technologies, (basically all innovation based on data, automation, artificial intelligence, predictive diagnostics, remote monitoring and analysis, personalised medicine, etc.) and therefore, will need to take account of the further development of those technologies. This will mean a riskbased policy with oversight proportionate to the intended use of the technology and led by defined risk categories [9].

(2) Support to innovation focussed on improved patient outcomes

The EHDS framework will need to be developed and implemented with an innovationsupportive approach. This will mean alignment with existing legislation in the area of data, and avoiding over-regulation with the right balance, promoting the highest level of trust and innovation in order to ensure early access for patients to healthcare innovation. Furthermore, the framework should guide new reimbursement and financing models needed to facilitate integrated digital healthcare and recognise the value of health data.

(3) Trust: working in the best interest of patients

None of the big health data transitions can happen without society's trust in the process. The EHDS has great potential to support ground-breaking innovation for patients, to enhance patient outcomes, increase healthcare equality and healthcare efficiencies. Those are the essential elements of the whole foundational process. Furthermore, responsible authorities need to invest in raising the levels of knowledge and awareness amongst patients, in order to increase trust, ensuring that they are active players in the process and increase understanding of their irreplaceable role in making the EHDS a reality. The authorities need to ensure that patients' concerns are addressed and that all data-sharing and data-usage models are built with the highest principles of ethics and transparency, to promote the altruistic sharing of data in the interest of the patient community and healthcare systems as a whole. The increased knowledge will, furthermore, empower patients and enable self-care.

(4) Better and interoperable data

In the digital and integrated care field, the results are only as good as the quality of the data generated. The adoption of the common electronic health record exchange format was very significant in the development of systems interoperability in healthcare. The European Commission acknowledges that interoperability can reduce costs, generated by uncoordinated approaches. A general line in the EHDS framework is that data needs to be in the same format and correspond to a common data quality, cyber security and other interoperability standards on which HCPs can rely. All stakeholders in the process need to be committed but also incentivised to transition to a fully interoperable health data environment.

(5) Digital health education

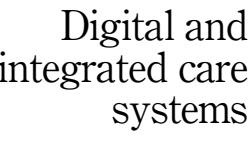

369 
JICA

30,4

Digitisation presents great demands on HCPs who are required to adapt their clinical pathways and processes. Promoting professional education and training towards digital literacy for HCPs must be an integral part of the policy agenda, (in university education and lifelong learning programmes). The EHDS initiative should as per the International Foundation for Integrated Care "enable individuals and the system to be their own change agents will create an environment that can effectively respond to the continuous evolution of communities and populations alongside being able to harness the potential of innovations and new ways of working" [10].

(6) The role of all players in the process

The perspectives of all concerned players will need to be taken into account otherwise the potential of the EHDS to contribute to a truly integrated healthcare system will not be realised. It is of course equally true that all players should contribute to the EHDS becoming a reality [11]:

- Public sector - with population health data and inter-government agreements;

- Research centres and biobanks - with research data and registries;

- Healthcare providers and professionals - with the setup of the electronic health records, hospital information, lab reports, real-world data;

- Industry - with registered information, clinical trials information;

- Payers - with health outcome information, cost/reimbursement information;

- Citizens and patients - with their real-world data, electronic health records and hospital information;

\section{Call to action and conclusion}

The COVID-19 pandemic has demonstrated the urgency of better chronic disease management and the importance of making it an integral part of the recovery agenda in Europe.

We believe that an orchestrated shift towards integrated care holds the solution to the chronic disease pandemic. The development of integrated care, however, cannot happen without shifting towards a digitalised healthcare system via large-scale initiatives like the EHDS and the involvement of all stakeholders.

Now is the right time to rethink healthcare systems in Europe, using the momentum of the COVID-19 pandemic and the inevitable and accelerating digitalisation in health. For that it is crucial to have the right healthcare systems set-up to provide digital solutions for patients to self-manage their chronic conditions and for HCPs to be able to support patients remotely. Such solutions would not be possible without the seamless generation and access to high quality, interoperable, integrated health data, precisely through initiatives like the EHDS.

The EGIDE group has identified some foundational principles, which can guide the way to realise the full potential of the EHDS for integrated care and can support the involved stakeholders in their thinking, and namely:

(1) Policy flexibility

(2) Support to innovation focussed on improved patient outcomes

(3) Trust: working in the best interest of patients

(4) Better and interoperable data 
(5) Digital health education

(6) The role of all players in the process

This reflection paper aims to serve as a starter of a constructive dialogue at various levels. We hope it is of value to the stakeholders who are advancing and benefiting from the EHDS initiative and who are looking at the re-organisation of the healthcare systems in Europe in general - patients, HCPs, EU and national policymakers, digital infrastructure players, EU and national implementing bodies, national insurers and the healthcare payer and research community.

\begin{abstract}
About the authors
The EGIDE, was brought together to contribute to filling a gap between the concept of integrated care and its implementation in Europe. The group was first established in 2019 with an aim to focus on diabetes, where members believed the greatest value could be achieved. Nonetheless, over the course of the COVID-19 pandemic, the focus of the group has shifted towards a broader approach to chronic diseases, and how to promote integrated care and its full potential to reverse the course of the chronic disease pandemic.

The members of this action-oriented group come from different backgrounds - patients, HCPs , policymakers, academics, industry representatives and former payers-and share one goal: to pool their knowledge, experience and understanding to propose specific policy solutions that will foster an environment where all aspects of chronic diseases are optimally and proactively integrated in order to measurably improve the lives of people with chronic diseases. The EGIDE group is most active in France, Germany, Ireland, Italy, the Netherlands, and Spain. Its work is supported by Sanofi.
\end{abstract}

\title{
Notes
}

1. https:/www.who.int/news-room/fact-sheets/detail/noncommunicable-diseases

2. https://www.researchgate.net/publication/346084372_Putting_people_at_the_centre_Integrated_ care_for_chronic_diseases_in_Europe_EGIDE_Policy_Paper_-_2020

3. https://alliancechronicdiseases.org/wp-content/uploads/ECDA-statement-on-COVID-19-chronicdiseases-and-EU-health-capacity-June-2020.pdf

4. https:/www.thelancet.com/journals/lancet/article/PIIS0140-6736(20)31067-9/fulltext

5. Integrated care models: an overview (who.int)

6. https://www.researchgate.net/publication/346084372_Putting_people_at_the_centre_Integrated_ care_for_chronic_diseases_in_Europe_EGIDE_Policy_Paper_-_2020

7. https://ec.europa.eu/health/ehealth/dataspace_en

8. https://www.medtecheurope.org/wp-content/uploads/2019/11/MTE_Nov19_AI-in-MedTechDelivering-on-the-Promise-of-Better-Healthcare-in-Europe.pdf

9. https://www.efpia.eu/media/554841/efpia-ehds-position_final.pdf

10. Workforce capacity and capability-IFIC (integratedcarefoundation.org)

11. 19106_COC_EU_Health_Data_SPACE_web.pdf (cocir.org)

\section{References}

Hazarika, I. (2020), "Artificial intelligence: opportunities and implications for the health workforce", Oxford Journals, Vol. 12 No. 4, pp. 241-245. 
JICA 30,4

Topol, C. (2019), Deep Medicine: How Artificial Intelligence Can Make Healthcare Human Again, Basic Books, New York.

\section{About the authors}

Stefano Genovese-Head of the Diabetology, Endocrinology and metabolic diseases in Milan Cardiological Centre and EGIDE Member, Italy.

Rafael Bengoa - Co-Director of the Bilbao Institute for Health and Strategy and EGIDE Member, Spain.

John Bowis-Former Minister of Health, UK, former Member of the European Parliament, person living with diabetes and EGIDE Co-Chair, UK.

Mary Harney-Former Minister of Health and Deputy Prime Minister, Ireland and EGIDE CoChair, UK.

Bastian Hauck-Director at the International Diabetes Federation Europe, Founder of \#dedoc ${ }^{\circ}$ Diabetes Online Community and EGIDE Member, Germany.

Michel Pinget-President of the CEED (European Centre for the Study of Diabetes), France.

Mike Leers-Former CEO, CZ Zorverzekeringen and EGIDE Member, The Netherlands.

Tarja Stenvall-Senior Vice President, Region Head of General Medicines Key Markets, SANOFI.

Nick Guldemond-Senior researcher at Leiden University Medical Center, Dept of Public Health and

Primary Care, National eHealth Living Lab (NeLL), Professor at I.M. Sechenov First Moscow State Medical university, Faculty of Preventive Medicine, Dept. of Public Health and Healthcare N.A. Semashko and EGIDE Member, The Netherlands. Nick Guldemond is the corresponding author and can be contacted at: nick@guldemondic.com

For instructions on how to order reprints of this article, please visit our website:

www.emeraldgrouppublishing.com/licensing/reprints.htm

Or contact us for further details: permissions@emeraldinsight.com 